\title{
Cultura organizacional a través de la gerencia educativa de una universidad privada en Perú
}

\author{
Organizational culture through the educational management of a private university in Peru
}

Cultura organizacional através da gestão educacional em uma universidade privada no Peru

ARTíCULO DE INVESTIGACIÓN

Rosa Diana Pedroza San Miguel

rosa.pedroza29@gmail.com

https://orcid.org/0000-0002-4473-8395

Universidad César Vallejo, Lima-Perú

Sara Pamela Sánchez Sandoval

pamelasanchezsan@gmail.com

https://orcid.org/0000-0002-6134-6908

Universidad César Vallejo, Lima-Perú
Luz Emerita Cervera Cajo

luz_cervera@hotmail.com https://orcid.org/0000-0003-1530-7761

Universidad César Vallejo, Lima-Perú

\section{Aura Elisa Quiñones Li}

auraelisa2006@hotmail.com

https://orcid.org/0000-0002-5105-1188

Universidad César Vallejo, Lima-Perú

Artículo recibido en junio 2021, revisado en julio 2021, arbitrado en agosto 2021 y publicado en septiembre 2021

\section{RESUMEN}

La cultura organizacional valora los principios que conforman la personalidad de una organización mediante sus procesos $\mathrm{y}$ actitudes. El objetivo planteado fue determinar como la cultura organizacional influye en el compromiso organizacional y la satisfacción laboral en colaboradores de una Universidad Privada. La metodología empleada fue un enfoque cuantitativo, correlacional, de diseño no experimental y de corte transversal, la muestra estuvo conformada por 132 docentes. Los resultados obtenidos demuestran que los colaboradores presentan una cultura organizacional de nivel alto a nivel general y por dimensiones; respecto al compromiso organizacional fue de nivel medio, mientras que, la satisfacción laboral es media con un $82,75 \%$. En relación con la influencia de la cultura organizacional en el compromiso organizacional y la satisfacción laboral, se observa una influencia positiva y significativa entre la cultura organizacional y la satisfacción laboral, pero no entre la cultura organizacional y el compromiso organizacional.

Palabras clave: Cultura organizacional; Productividad laboral; Satisfacción laboral; Habilidades profesionales

\section{ABSTRACT}

Organizational culture values the principles that shape the personality of an organization through its processes and attitudes. The objective was to determine how organizational culture influences organizational commitment and job satisfaction in employees of a private university. The methodology used was a quantitative, correlational, non-experimental design and cross-sectional approach, the sample consisted of 132 teachers. The results obtained show that the collaborators present a high level organizational culture at a general level and by dimensions; with respect to organizational commitment it was of medium level, while job satisfaction is medium with $82.75 \%$. In relation to the influence of organizational culture on organizational commitment and job satisfaction, a positive and significant influence was observed between organizational culture and job satisfaction, but not between organizational culture and organizational commitment.

Key words: Organizational culture; Job productivity; Job satisfaction; Professional skills

\section{RESUMO}

A cultura organizacional valoriza os princípios que moldam a personalidade de uma organização através de seus processos e atitudes. O objetivo era determinar como a cultura organizacional influencia o compromisso organizacional e a satisfação no trabalho dos funcionários de uma universidade privada. A metodologia utilizada foi uma concepção quantitativa, correlacional, não experimental, transversal, e a amostra consistiu de 132 professores. Os resultados obtidos mostram que os funcionários têm um alto nível de cultura organizacional em nível geral e por dimensões; com relação ao compromisso organizacional foi médio, enquanto a satisfação no trabalho é média, com $82,75 \%$. Em relação à influência da cultura organizacional no compromisso organizacional e na satisfação no trabalho, observou-se uma influência positiva e significativa entre cultura organizacional e satisfação no trabalho, mas não entre cultura organizacional e compromisso organizacional.

Palavras-chave: Cultura organizacional; Produtividade no trabalho; Satisfação no trabalho; Habilidades profissionais; Satisfação no trabalho 


\section{INTRODUCCIÓN}

Una organización exitosa cuenta con los mejores colaboradores, los cuales son la clave para la sostenibilidad de cualquier organización (Peña, et al., 2016). Por ello, la gestión de los colaboradores es clave, en el reclutamiento y selección de personal, pero sobre todo en el transcurso de la formación y adaptación del personal, el cual permite crear un sentido de pertenencia y compromiso entre los equipos de colaboradores y la institución (Esan, 2015). El Índice de Compromiso Global (GEI) muestra que son pocos los empleados que están verdaderamente comprometidos en la organización o institución en relación al trabajo que realizan.

El compromiso y la cultura son los principales factores a adoptar en cualquier organización del planeta. El entorno se ha vuelto más competitivo, por lo que, se han creado nuevas formas de hacer las cosas, como modificar la cultura que afecta el comportamiento organizacional (Cifuentes y Londoño, 2017). En este contexto, diversos estudios han demostrado que el elevar el compromiso laboral de los colaboradores en la organización, es la mejor forma de incrementar su productividad y aumentar su bienestar en el trabajo (Calderón, 2016). El aporte de los colaboradores en las actividades y la actitudes positivas hacia su trabajo está en función de la percepción que estos tienen de la organización (Fonseca, y Cruz, 2019). Según Goleman, la satisfacción depende fundamentalmente de las emociones que las personas experimentan en el lugar de trabajo (Rojas, 2017). En este sentido, la cultura organizacional ha mostrado un impacto directo en la satisfacción de los empleados y el compromiso organizacional (Tinoco, Quispe, y Beltrán, 2014).

Continuando bajo este orden de ideas, se tiene que las instituciones educativas no son inmunes a los cambios y desafíos actuales. Las nuevas necesidades educativas requieren mejores destrezas y habilidades profesionales, así como la adaptabilidad a los nuevos horarios y funciones, lo que requiere cambiar el sistema organizacional tradicional (Concha, 2016). Además, uno de los problemas que suelen surgir en las instituciones educativas es que algunos de sus trabajadores no llevan a cabo el sentido de pertenencia para emplear el valor dentro de la institución donde se labora, por lo que no se han formado compromisos reales y permanentes, y se ha mantenido la tasa de rotación de personal docente alta (Rojas y Távara, 2017).

La satisfacción laboral es un problema global de salud pública que afecta a todas las organizaciones, debido a la alta tasa de insatisfacción de los colaboradores, frustra su relación laboral, amical y familiar afectando significativamente en su desempeño laboral (Zelada, 2018). En el sector educación, los maestros son colaboradores de las organizaciones de educación, por lo que, si el clima organizacional es positivo o negativo, esto se reflejará en su comportamiento. Las investigaciones realizadas en universidades privadas pueden asegurar que el entorno laboral se relaciona con la conducta de los docentes, y ello se refleja en su desempeño en el aula. Esto es importante porque los resultados se reflejan en el crecimiento de los estudiantes (Picoagua, 2018).

En Perú actualmente las universidades mantienen una alta tasa de crecimiento, cada año se crean más universidades (Aquino, 2018). Esto lleva a reflexionar, si estas instituciones tienen una cultura organizacional que propicie a los docentes y otros colaboradores a comprometerse con la organización, y tratar de potenciar la satisfacción de todos los colaboradores. En ese sentido, el estudio e intervención en estos temas se hace necesario en el proceso de mejoramiento de la institución y formación de los futuros profesionales del país. 
El estudio se justifica a nivel teórico, porque las variables se sustentan en la literatura consultada. En este sentido, en cuanto a las variables de cultura organizacional, es uno de los ejes básicos, por lo cuales las entidades pueden ser más competitivas sin dejar de lado el compromiso organizacional, el cual se basa en la psicología organizacional, especialmente en el ámbito humano. En toda organización, el rol de los colaboradores es esencial, por lo que el instrumento de medición se basa en la teoría de Meyer y Allen (1991). Para evaluar la satisfacción laboral se consideró la literatura de (Robbins, 2005). Todos estos procesos pueden servir de referencia para otras encuestas que involucran cultura organizacional, compromiso organizacional y satisfacción laboral. Finalmente, su propósito es que los empleadores comprendan y evalúen estas dos variables interrelacionadas e interactuantes, porque los resultados ayudan a fortalecer la organización en relación con los tópicos estudiados. Esta investigación tiene una justificación práctica, porque estas variables son aplicables a métodos de comportamiento humano, entre los cuales se prioriza a los colaboradores, por lo que los resultados de este estudio favorecen a la universidad privada de Los Olivos.

El estudio tiene como objetivo determinar si la cultura organizacional influye en el compromiso organizacional y la satisfacción laboral en colaboradores de una Universidad Privada del distrito de Los Olivos en el año 2021.

\section{MÉTODO}

La investigación fue de tipo descriptivo y de alcance correlacional, de corte transversal. La población estuvo conformada por 200 colaboradores de Universidades privadas del Distrito de Los Olivos, y la muestra se calculó mediante la fórmula estadista para poblaciones finitas, obteniendo una muestra conformada por 132 colaboradores. La técnica empleada fue la encuesta y se utilizaron 3 cuestionarios por cada variable estudiada, el primero de ello fue cuestionario de cultura organizacional en donde se desarrollaron cuatro (4) dimensiones: cultura orientada al poder, cultura orientada al rol, cultura orientada a la tarea y cultura orientada a la persona. El segundo instrumento fue sobre Compromiso Organizacional compuesto por tres (3) dimensiones: afectivo, continuidad y normativo; el ultimo cuestionario fue acerca de la satisfacción laboral, el cual estuvo compuestos por cuatro (4) dimensiones: reto del trabajador, remuneraciones justas, condiciones de trabajo y relación trabajoempleado; todos los instrumentos aplicados fueron validados previamente por expertos.

Luego de solicitar los permisos correspondientes se aplicaron los instrumentos, y a partir de la información recabada, se organizaron los datos en Excel, teniendo en consideración todos los datos y procedimientos. Posteriormente se analizaron los datos en un software estadístico utilizado por las ciencias sociales (IBM SPSS Statistics) en su versión 25 en español. El análisis descriptivo, los datos se agruparon en frecuencias numéricas y porcentuales por variables y dimensiones, por lo tanto, la frecuencia permitió conocer los niveles de sus variables en forma absoluta y porcentual. Así mismo, los datos obtenidos permitieron determinar los niveles de confiabilidad de los instrumentos mediante una prueba piloto, el estadístico utilizado fue Alfa de Cronbach.

Respecto al análisis inferencial, se procedió a comprobar las hipótesis de forma estadística, con el fin de determinar la existencia o no de correlación y su nivel de significancia, para ello en primer lugar se procedió a realizar una prueba de normalidad o bondad de ajuste a fin de poder determinar si el comportamiento de los datos tiene o no normalidad y con ello seleccionar estadísticamente una prueba paramétrica o no paramétrica. 
RESULTADOS Y DISCUSIÓN

Con respecto a los resultados descriptivos se obtuvo lo siguiente:

Se observa en la Tabla 1 que la totalidad de los colaboradores encuestados manifestaron que existe una cultura organizacional de nivel alto. En todas las dimensiones de la variable cultura, la cultura orientada al poder, al rol, a la tarea y a la persona, perciben los colaboradores un nivel alto.

Tabla 1. Cultura Organizacional por dimensiones.

\begin{tabular}{lccc}
\hline Dimensiones & Nivel & Frecuencia & Porcentaje \\
\hline Cultura orientada al poder & Alto & 100 & $100,0 \%$ \\
Cultura orientada al rol & Alto & 100 & $100,0 \%$ \\
Cultura orientada a la tarea & Alto & 100 & $100,0 \%$ \\
Cultura orientada a la persona & Alto & 100 & $100,0 \%$ \\
\hline
\end{tabular}

Seguidamente en la Tabla 2 se observa que el $87 \%$ de los colaboradores encuestados manifestaron que existe un compromiso organizacional de nivel medio. En la dimensión afectivo el 39\% consideró que existe un nivel alto, y $61 \%$ un nivel medio, mientras que, en las dimensiones continuidad y normativo el $100 \%$ considera un nivel medio.

Tabla 2. Compromiso Organizacional por dimensiones.

\begin{tabular}{lccc}
\hline Dimensiones & Nivel & Frecuencia & Porcentaje \\
\hline \multirow{2}{*}{ Afectivo } & Medio & 61 & 39 \\
\multirow{2}{*}{ Continuidad } & Alto & 100 & $39,0 \%$ \\
Normativo & Medio & 100 & $100,0 \%$ \\
\hline
\end{tabular}

En la Tabla 3 se puede observar que el $82.75 \%$ de los colaboradores encuestados manifestaron que existe una satisfacción laboral de nivel medio. En la dimensión remuneraciones justas el 31\% considera que existe un nivel alto y $69 \%$ un nivel medio, mientras que, en las dimensiones reto del trabajo, condiciones de trabajo y relación trabajo-empleo, el $100 \%$ considera un nivel alto. 
Tabla 3. Satisfacción Laboral por dimensiones.

\begin{tabular}{lccc}
\hline Dimensiones & Nivel & Frecuencia & Porcentaje \\
\hline Reto del trabajo & Alto & 100 & $100,0 \%$ \\
& Medio & 69 & $69,0 \%$ \\
Remuneraciones justas & Alto & 31 & $31,0 \%$ \\
Condiciones de trabajo & Alto & 100 & $100,0 \%$ \\
Relación trabajo - empleado & Alto & 100 & $100,0 \%$ \\
\hline
\end{tabular}

Con respecto a los resultados inferenciales se obtuvo:

\section{Hipótesis general}

Se obtuvo que la cultura organizacional y la satisfacción laboral obtienen un coeficiente Rho de Spearman $=0,911$, con un grado de significancia $p=0,000$, se rechaza la premisa nula y se acepta la premisa alterna; la cultura organizacional influye positiva y de manera significativa en la satisfacción laboral de los colaboradores de una Universidad Privada del distrito de los Olivos. Por otro lado, al correlacionar las cambiantes cultura y el compromiso organizacional se recibe un coeficiente Rho de Spearman $=0,004$, con un grado de significancia $p=0,971>0,05$, entonces se rechaza la premisa alterna y se acepta la premisa nula; la cultura organizacional no influye en el compromiso organizacional de los colaboradores de una Universidad Privada del distrito de los Olivos. Al final, se establece que la cultura organizacional influye de manera significativa en la satisfacción laboral, sin embargo, no influye sobre el compromiso laboral de los colaboradores.

\section{Hipótesis específica 1}

Se obtuvo que la cultura organizacional y el compromiso afectivo obtienen un coeficiente Rho de Spearman $=0,012$, con un grado de significancia $\mathrm{p}=0,907>0,05$, entonces se rechaza la hipótesis alterna y se acepta la hipótesis nula; la cultura organizacional no influye sobre el compromiso afectivo de los trabajadores de una Universidad Privada del distrito de los Olivos. Asimismo, al correlacionar las cambiantes cultura organizacional y la interacción de trabajo-empleado se recibe un coeficiente Rho de Spearman $=-0,490$, con un grado de significancia $\mathrm{p}=0,000$, por lo que se rechaza la hipótesis nula y se acepta la hipótesis alterna; la cultura organizacional influye negativa y de manera significativa en la interacción de trabajo-empleado de los colaboradores de una Universidad Privada del distrito de los Olivos. Al final, se establece que la cultura organizacional influye significativa $y$ de manera negativa en la interacción de trabajoempleado, pero no influye sobre el compromiso afectivo de los colaboradores.

\section{Hipótesis específica 2}

Se obtuvo que la cultura y el compromiso de continuidad obtienen un coeficiente Rho de Spearman $=-0,026$, con un nivel de significancia $\mathrm{p}=$ $0,800>0,05$, entonces se rechaza la hipótesis alterna y se acepta la hipótesis nula; la cultura organizacional no influye sobre el compromiso continuidad de los colaboradores de una Universidad Privada del distrito de los Olivos. Asimismo, al correlacionar las variables cultura organizacional y remuneraciones 
justas se obtiene un coeficiente Rho de Spearman $=0,122$, con un nivel de significancia $\mathrm{p}=0,227>$ 0,05 , entonces se rechaza la hipótesis alterna y se acepta la hipótesis nula; la cultura organizacional no influye en las remuneraciones justas de los colaboradores de una Universidad Privada del distrito de los Olivos. Finalmente, se determina que la cultura organizacional no influye en el compromiso continuidad y remuneraciones justas en colaboradores.

\section{Hipótesis específica 3}

Se obtuvo que la cultura y el compromiso normativo obtienen un coeficiente Rho de Spearman $=-0,067$, con un nivel de significancia $\mathrm{p}=0,509>0,05$, entonces se rechaza la hipótesis alterna y se acepta la hipótesis nula; la cultura organizacional no influye en el compromiso normativo de los colaboradores de una Universidad Privada del distrito de los Olivos. Por el contrario, al correlacionar las variables cultura organizacional y condiciones de trabajo se obtiene un coeficiente Rho de Spearman $=0,838$, con un nivel de significancia $\mathrm{p}=0,000<0,01$, entonces se rechaza la hipótesis nula y se acepta la hipótesis alterna; la cultura organizacional influye positiva $y$ significativamente en las condiciones de trabajo de los colaboradores de una Universidad Privada del distrito de los Olivos. Finalmente, se determina que la cultura organizacional influye significativamente en las condiciones de trabajo, pero no influye en el compromiso normativo de los colaboradores.

\section{Discusión}

El estudio planteó como objetivo determinar cómo la cultura organizacional influye en el compromiso organizacional y la satisfacción laboral en colaboradores de una Universidad Privada del distrito de Los Olivos. Los resultados muestran una influencia directa y significativa de la cultura en la satisfacción laboral, pero no se evidencia relación entre la cultura y el compromiso; según Cuenca y López (2020) la cultura organizacional influye de forma moderada pero significativa en el compromiso de los colaboradores de una institución, sin embargo, consideran que para que esta relación sea más fuerte, se requiere de estrategias que eleven el apego de los colaboradores con la organización. El apego genera actitud de compromiso, un compromiso continuo mejora la relación y el desempeño de los colaboradores con la organización (Bayona, et al., 2015; Fuentes, 2018).

El compromiso organizacional está asociado a factores como la comunicación interna, los intereses de gestión, la formación y el equilibrio trabajofamilia (Ruiz, 2013); estos factores sobre todo en el ámbito educativo motivan a los colaboradores a identificarse con su institución y a trabajar de manera conjunta para lograr los objetivos organizacionales (Hernández et al., 2018). En la institución estudiada, los colaboradores presentan un nivel de mediano a alto en todas las dimensiones de la variable compromiso (afectivo, continuidad y normativo), que no han sido producto de la excelente cultura organizacional percibida por los miembros encuestados; aunque la cultura está fuertemente asociada a la motivación en el trabajo (Aguirre, 2017), un estilo de liderazgo y la satisfacción laboral podrían impactar de forma más significativa en el compromiso organizacional (Guerrero, 2016).

La cultura organizacional se presentó en un nivel alto en todos los colaboradores encuestados y en cada una de las dimensiones estudiadas (cultura organizacional orientada al poder, al rol, a la tarea 
y a la persona), lo cual no significó una ventaja para mejorar el compromiso en ninguna de sus dimensiones (afectivo, de continuidad y normativo), ni en las remuneraciones justas que perciben los colaboradores; contradiciendo lo manifestado por Lupano y Castro (2019), que consideran que las virtudes organizacionales, entre ellos la cultura, tienen un impacto directo en el grado de satisfacción y de compromiso en la organización. No obstante, Wills et al., (2017) afirman que, el sentido de pertenencia de los empleados con la organización es más fuerte si los empleados están satisfechos con la comunicación interna, y por ende desarrollan un mayor compromiso laboral.

La cultura organizacional promueve mejores condiciones laborales y una saludable relación trabajo-empleado. Las condiciones de trabajo, la relación trabajo-empleado y el reto de trabajo, se muestran en un nivel alto en todos los colaboradores en estudio, mientras que, se observa un nivel medio de satisfacción laboral y de percepción de remuneraciones juntas; todos estos factores en conjunto contribuyen a una mejor satisfacción en el trabajo, satisfacción que al igual que el compromiso mejoran el desempeño laboral y permiten el cumplimiento de los objetivos organizacionales (Vargas y Flores, 2019; Guerrero, 2016; Pedraja et al., 2020).

Los hallazgos presentados y los antecedentes revisados muestran que existe una fuerte asociación entre las variables cultura organizacional $y$ satisfacción laboral, sin embargo, no existe mucha literatura que demuestre una significativa relación de la cultura con el compromiso laboral, pero si la variable liderazgo se relaciona con el compromiso organizacional, así como la satisfacción y el compromiso con el desempeño laboral (Fuentes,
2018; Lomas, 2017; Salvador y Sánchez, 2018; Pedraza, 2020). Asimismo, la motivación laboral es esencial para elevar el compromiso de los colaboradores con su labor (Puma, 2020).

\section{CONCLUSIONES}

La cultura organizacional influye directa y significativamente en la satisfacción laboral pero no influye en el compromiso organizacional dentro de la universidad objeto de estudio, también se pudo determinar que la cultura organizacional influye de manera moderada e inversa en la relación de trabajo empleado, pero no influye en el compromiso afectivo en colaboradores, también fue determinado que la cultura organizacional no influye en las dimensiones de compromiso de continuidad, ni en las remuneraciones justas, para finalizar se puede concluir que la cultura organizacional influye de manera directa y significativa en las condiciones de trabajo, pero no influye en el compromiso normativo en colaboradores de una Universidad Privada del distrito de Los Olivos.

\section{REFERENCIAS}

Aguirre, P. (2017). Cultura organizacional y motivación laboral de los trabajadores de la Unidad de Gestión Educativa Local No 04 - 2016. Lima, Perú. (Tesis de maestría). Universidad Cesar Vallejo

Aquino, R. (2018). Compromiso Organizacional en la Universidad Privada de Huancayo. Huancayo, Perú. Obtenido de http:// repositorio.upla.edu.pe/bitstream/handle/ U P L A / $718 /$ T 037 _ 40683928 _ T. pdf? sequence $=1$ yisAllowed $=y$

Bayona, C., Goñi, S., y Madorrán, C. (2015). Compromiso Organizacional: Implicaciones para la gestión estratégica de los Recursos. 
Revista Europea de Direccion y Economia de la Empresa, 1-25.

Calderón, J. (2016). Socialización y compromiso organizacional: una revisión a partir del bienestar laboral. Redalyc, 239-247. Obtenido de https:// www.redalyc.org/pdf/292/29248182003.pdf

Cifuentes, L., y Londoño, M. (2017). Rasgos Identificativos que promueven la Cultura de la Innovación en las. Revista Interamericana de psicología ocupacional, 33-48. doi:10.21772/ ripo. v36n1a03

Concha, A. (2016). RELACIÓN ENTRE LA SATISFACCIÓN LABORAL CON LA COMUNICACIÓN INTERNA. Guayaquil. Obtenido de http://200.31.31.137:8080/ bitstream/ucasagrande/1049/1/Tesis1222CONr. pdf

Cuenca, R., y López, H. (2020). Estudio de la gestión del compromiso y cultura organizacional de FLACSO. Podium, (37),43-56. doi: https://doi. org/10.31095/podium.2020.37.4

Esan. (22 de Mayo de 2015). La importancia del capital humano para el éxito en los negocios. Obtenido de conexionesan: https://www. esan.edu.pe/apuntes-empresariales/2015/05/ importancia-capital-humano-para-exitonegocios/

Fonseca, J., y Cruz, C. (2019). Relación del Bienestar Subjetivo de los trabajadores en su compromiso hacia la organización. CES Psicología, 126-140. doi:http://dx.doi.org/10.21615/cesp.12.2.9

Fuentes, K (2018). Compromiso organizacional $y$ desempeño laboral en los trabajadores de la Unidad de Gestión Educativa Local 01 - San Juan de Miraflores, 2018. Lima- Perú. (Tesis para optar el título de maestría). Universidad Cesar Vallejo.

Guerrero, M. (2016). La relación entre los estilos de liderazgo, la satisfacción laboral y su efecto en el compromiso organizacional. Uid, 134-144. doi: https://doi.org/10.33890/innova.v1.10.2016.106

Hernández, B., Ruiz, A., Ramiírez, V., Sandoval, S., y Méndez, L. (2018). Motivos y factores que intervienen en el compromiso organizacional. Ride. doi: 10.23913/ride. v8i16.370
Lomas, R. (2017) Cultura organizacional y motivación laboral de los trabajadores de la Unidad de Gestión Educativa Local No 04 2016. Lima -Peru. (Tesis para optar el título de maestra). Universidad Cesar Vallejo.

Lupano, M., y Castro, A. (2019). Influencia de virtudes organizacionales sobre satisfacción, compromiso y performance laboral en organizaciones argentinas. Redalyc, 171-188.

Meyer, J. y Allen, N. (1991). A three-component conceptualization of organizational commitment. Human Resource Management Review, 1, 61- 89. http://dx.doi.org/10.1016/10534822(91)90011-Z

Pedraja, L., Marchioni, I., Espinoza, C., y Muñoz, C. (2020). Liderazgo y cultura organizacional como factores de influencia en la calidad universitaria: un análisis conceptual. Form. Univ., 13(5), 3-14. doi: http://dx.doi.org/10.4067/S071850062020000500003

Pedraza, N. (2020). Satisfacción laboral y compromiso organizacional del capital humano en el desempeño en instituciones de educación superior. RIDE. Rev. Iberoam. Investig. Desarro. Educ., 10(20), 1-29.doi: https://doi. org/10.23913/ride.v10i20.595

Peña, M., Díaz, G., Chávez, A., y Sánchez, C. (2016). El compromiso organizacional como parte del comportamiento de los trabajadores de las pequeñas empresas. Revista Internacional Administración y Finanzas, 95-105.

Picoagua, J. (2018). Clima Organizacional y Satisfacción laboral según Docentes de una Universidad Privada. Lima, Perú. Obtenido de http://repositorio.uwiener.edu.pe/bitstream/ handle/123456789/2543/TESIS\%20Picoaga\%20 Jos\%c3\%a9.pdf? sequence $=1$ yisAllowed $=y$

Puma, M. (2020). La motivación laboral y el compromiso organizacional. Ciencia $y$ Desarrollo, 23(3), 45-53. doi: http://dx.doi. org/10.21503/cyd.v23i3.2141

Robbins, S. (2005). Administración ( $8^{\circ}$ ed.). México: Pearson educación.

Rojas, N. (2017). Satisfacción laboral e incidencia en el compromiso organizacional de docentes de 
centros de educación básica alternativa del ciclo avanzado de la región Puno. Revista Científica "Investigación Andina", 14-20. Obtenido de https://revistas.uancv.edu.pe/index.php/RCIA/ article/view/352/294

Rojas, W., y Távara, U. (2017). Cultura organizcional y satisfacción laboral en los trabajadores de la universidad Marcelino Champagnat. Lima, Perú. Obtenido de http://repositorio.umch.edu. pe/bitstream/UMCH/113/1/35.\%20Tesis\%20 $\% 28$ Rojas $\% 20$ V \% c $3 \%$ a 1 squez $\% 20$ y $\% 20$ T\%c3\%alvara\%20Zegarra\%29.pdf

Ruiz, J. (2013). El compromiso organizacional: un valor personal y empresarial en el marketing interno. Revista de Estudios Empresariales. Segunda época, 67-86. Recuperado: https:// dialnet.unirioja.es/descarga/articulo/5089649

Salvador, E.M., y Sánchez, J.A. (2018). Liderazgo de los directivos y compromiso organizacional Docente. Rev. investig. Altoandin. 20 (1), 115-124. doi: http://dx.doi.org/10.18271/ria.2018.335

Tinoco, O., Quispe, C., y Beltrán, V. (2014). Cultura organizacional y satisfacción laboral en la facultad de Ingeniería Industrial en el marco de la acreditación universitaria. Revista de la Facultad de Ingeniería Industrial, 56-66.
Vargas, S., y Flores, M. (2019). Cultura organizacional y satisfacción laboral como predictores del desempeño laboral en bibliotecarios. Bibliotecológica, 33 (79), 149-176. Recuperado de http://www.scielo.org.mx/pdf/ ib/v33n79/2448-8321-ib-33-79-149.pdf

Wills, N., Cevallos, M., Sadi, G., y Ancin, I. (2017). La relación entre la satisfacción con la comunicación interna y el compromiso organizacional: el caso de una universidad ecuatoriana. Austral Comunicación, 6 (1), 133160. Recuperado:https://dialnet.unirioja.es/ servlet/articulo? codigo $=6067433$

Zelada, E. (2018). La Gestión del talento humano y la Satisfacción laboral en los Docentes de la Universidad Tecnológica del Perú. Obtenido de http://repositorio.ucv.edu.pe/ bitstream/handle/UCV/17305/Zelada_FEA. pdf? sequence $=1$ yisAllowed $=y$ 